\title{
Acute Colonic Volvulus in a Mexican Population: A Case Series
}

\author{
Mauricio Gonzalez-Urquijo ${ }^{1,2}$, Mario Rodarte-Shade ${ }^{1,2}$, Gerardo Gil-Galindo ${ }^{2}$ \\ ${ }^{1}$ Tecnologico de Monterrey, Escuela de Medicina y Ciencias de la Salud, Monterrey; ${ }^{2}$ Hospital Metropolitano "Dr. Bernardo Sepúlveda," \\ Secretaria de Salud de Nuevo León, San Nicolás de los Garza, México
}

Purpose: Colonic volvulus constitutes a significant cause of large bowel obstruction in adults. Most studies of colonic volvulus come from high endemic zones and are limited by the small number of patients. In our region, there is a shortage of studies concerning this disorder, and treatment of colonic volvulus remains controversial.

Methods: This is a retrospective study of 34 patients who presented with colonic volvulus at a single academic institution in a 4 -year period and their respective treatment and outcomes.

Results: A total of 34 patients, 17 males (50\%) and 17 females (50\%), with a mean age of $55 \pm 23.9$ years underwent treatment for colonic volvulus. Twelve patients (35.3\%) underwent initial decompression, followed by a Hartman procedure in 4 patients $(11.7 \%)$ and sigmoid resection with primary anastomosis in 3 patients $(8.8 \%)$, with 3 fatalities (8.8\%) following initial decompression. Two patients (5.8\%) were lost to follow-up. Twenty-two patients (64.7\%) underwent emergency surgery, of whom 16 (47\%) underwent a Hartman procedure, with colorectal anastomosis in 9 patients (26.4\%), with 3 fatalities (8.8\%) immediately after the first procedure. Four patients (11.7\%) were lost to follow up after the Hartman procedure. Of the 6 remaining patients (17.6\%), of the emergency surgical group, 3 patients (8.8\%) had an initial sigmoidectomy and primary anastomosis, and the remaining 3 patients $(8.8 \%)$ had a cecal volvulus with a right hemicolectomy performed with primary anastomosis in 2 patients (5.8\%) and with a fatality in the remaining patient, on whom a terminal ileostomy was performed for damage control. The mean hospital stay was 5.7 days, with an overall mortality rate of $23.5 \%$. Conclusion: Acute colonic volvulus in our region is not as uncommon as in other parts of the world. This disorder must be suspected when a patient presents with abdominal pain, abdominal distension, and bean sign on plain X-rays and/or a whirl sign on computed tomography scan.

Keywords: Colonic volvulus; Large bowel obstruction; Decompression; Sigmoid volvulus; Cecal volvulus

\section{INTRODUCTION}

Colonic volvulus refers to torsion of the bowel around its own mesentery $[1,2]$. This condition constitutes the third leading cause of large bowel obstruction in adults, following colorectal

Received: October 19, 2018 - Accepted: January 2, 2019

Correspondence to: Mauricio Gonzalez-Urquijo, M.D.

Tecnologico de Monterrey, Escuela de Medicina y Ciencias de la Salud, Dr. Ignacio Morones Prieto 0 3000, Monterrey 64710, México

Tel: +81-8888-0575

E-mail:mauriciogzzu@gmail.com

ORCID: https://orcid.org/0000-0001-5101-1541

(C) 2020 The Korean Society of Coloproctology

This is an open-access article distributed under the terms of the Creative Commons Attribution NonCommercial License (https://creativecommons.org/licenses/by-nc/4.0) which permits unrestricted noncommercial use, distribution, and reproduction in any medium, provided the original work is properly cited. cancer and complicated sigmoid diverticulitis, accounting for $3.4 \%$ of all bowel obstructions and $10 \%-30 \%$ of all colonic obstructions [1, 3-8]. The main predisposing factor to colonic volvulus is a long, redundant colon with a stretched mesentery, which is liable to become entangled [3]. Other predisposing factors include a long history of poor bowel habits and chronic constipation with frequent use of laxative substances [9].

In the Middle East, sigmoid volvulus is 4 times as common as in Western countries. This may be due to a diet high in vegetables and endemic infections. Previous abdominal surgery, pregnancy, high altitude, pelvic tumors or cysts, and megacolon have all been reported in association with this condition $[1,3,6,9,10]$. Sigmoid volvulus occurs more commonly in the elderly, particularly in patients with dementia or psychiatric illness, $[3,4,9]$ and its presentation has high mortality not only because of associated medical 
problems, but also due to delay in presentation and a consequently higher incidence of ischemic bowel $[11,12]$.

We report the results of a series of 34 adult patients with colonic volvulus who underwent emergent treatment in an academic public institution over a 4-year period.

\section{METHODS}

All consecutive patients admitted with a colonic volvulus between 2014-2017 in our institution were included. Hospital records were retrospectively reviewed. All patients were admitted to our hospital on an emergency basis due to bowel obstruction. The following parameters were assessed: age, sex, symptoms and signs, diagnostic modalities employed (abdominal radiographs in erect and supine positions and/or computed tomography [CT] scan with or without IV contrast), hours from first symptom to admission, comorbidities, primary procedure (either surgical decompression or colonoscopic detorsion) with or without a secondary procedure (Hartman procedure or sigmoidectomy with colorectal anastomosis), emergent surgery (Hartman procedure or sigmoidectomy with colorectal anastomosis), length of hospital stay, laboratory results, outcomes, and mortality.

The patients were resuscitated and underwent correction of electrolyte deficits when necessary. A nasogastric tube was routinely used in all cases to decompress the small bowel at arrival at the emergency room. If a sigmoid detorsion was performed successfully, an 18F nelaton tube was placed in the rectum, and patients were prepared for elective sigmoid resection during the same hospitalization, with or without primary anastomosis. Surgical treatment was performed if the patient was admitted during the night shift, if endoscopy was not available, if the mucosa was necrotic and gangrenous during colonoscopy, or if the colonic volvulus involved the cecum.

During surgery for sigmoid volvulus, the descending colon and proximal rectum were mobilized, their vascularity was ensured, and a sigmoid resection and 2-layered anastomosis were carried out (sigmoidectomy + colorectal hand-sewn anastomosis). If the sigmoid colon was gangrenous, it was resected without untwisting, closing the rectal stump, and exteriorizing a colostomy on the left flank (Hartman procedure). At the end of both procedures, the peritoneal cavity was lavaged with warm normal saline. During surgery for cecal volvulus, the right colon was mobilized, separating it from the hepatic flexure, performing an isoperistaltic right hemicolectomy with a hand-sewn latero-lateral ileo transverse anastomosis. When anastomosis could not be achieved, a terminal ileostomy was performed, resecting the ileum at $10 \mathrm{~cm}$ from the ileocecal valve, exteriorizing through the right flank, and exteriorizing a mucous fistula.

Informed consent for volvulus detorsion or laparotomy and participation in surveillance protocols was obtained from all patients. Follow-up was conducted either by telephone or by consult after discharge at 1 month, 6 months, and yearly after the first year.
This study was approved by the Tecnologico de Monterrey Institutional Review Board under the number 043.

Data analysis was performed with a combination of Excel 16.0 (Microsoft Corp., Redmond, WA, USA) and R 3.4 (R Core Team, Auckland, New Zealand), using frequency measures (absolute and percentage) for categorical variables and mean \pm standard deviation and ranges for quantitative measures.

\section{RESULTS}

In this study, 34 patients with a mean age of $55 \pm 23.9$ years (range, 19-87 years) were treated, 17 (50\%) were male and 17 (50\%) were female. There were 11 patients (32.4\%) under 40 years old and 23 patients $(67.6 \%)$ over 40 . There were 31 patients $(91.2 \%)$ with sigmoid volvulus, 3 of whom presented with perforation, and only 3 patients (8.8\%) had cecal volvulus. Two-thirds of the patients had existing comorbidities (Table 1). In 4 patients (11.7\%), there were previous episodes of sigmoid volvulus diagnosed with CT scan prior to the study period.

The interval between development of symptoms and hospitalization ranged from 1 to 14 days (mean, $5.67 \pm 4.46$ days). Clinical manifestations included abdominal distension, abdominal pain, nausea, vomiting, diarrhea, and melena (Table 2). Abdominal examination revealed marked abdominal distension in all patients (100\%), with abdominal tenderness in 17 patients (50\%). Following rectal examination, blood on the examining finger was present in 3 patients (8.8\%). During the diagnostic stage, all 34 patients (100\%) underwent plain abdominal X-rays, with positive air-fluid levels and a coffee bean or omega sign in 26 patients (76.4\%), with a mean measurement of the widest intestinal loop of $13.1 \pm 3.87 \mathrm{~cm}$. Six patients (17.6\%) had only air-fluid levels,

Table 1. Patient comorbidities

\begin{tabular}{ll}
\hline Comorbidities & No. (\%) \\
\hline Chronic constipation & $7(20.6)$ \\
Psychiatric illness & $6(17.6)$ \\
Systemic arterial hypertension & $5(14.7)$ \\
Diabetes mellitus type II & $3(8.8)$ \\
Hypothyroidism & $1(2.9)$ \\
Cardiac failure & $1(2.9)$ \\
\hline
\end{tabular}

Table 2. Clinical manifestations

\begin{tabular}{lc}
\hline Symptoms & No. of patients \\
\hline Abdominal distension, abdominal pain, nausea and vomiting & $19(55.8)$ \\
Abdominal distension, abdominal pain, diarrhea & $5(14.7)$ \\
Abdominal distension, abdominal pain & $5(14.7)$ \\
Melena & $3(8.8)$ \\
Nausea and vomiting & $2(5.8)$ \\
\hline
\end{tabular}


with marked dilatation of the small bowel, and 2 patients $(5.8 \%)$ had radiological signs of bowel perforation. A CT scan of the abdomen was performed in 20 patients (58.8\%) with a dilated sigmoid colon; a whirl pattern was present in the mesentery in the left of midline in 13 patients (38.3\%), suggestive of sigmoid volvulus. In 3 patients (8.8\%), this sign was located to the right of midline, suggestive of the cecal counterpart. In 2 patients (5.8\%), CT revealed only dilated colonic loop, with a negative whirl sign. Blood chemistry and hematological profile were routinely studied. Mean values are displayed in Table 3.

After early and effective resuscitation, to complete decompression and derotation, colonoscopy or laparotomy with manual decompression was performed in 8 (23.5\%) and 4 patients $(11.7 \%)$, respectively, with fixation of a rectal tube at the end of the procedure. Of these 12 patients (35.2\%), 7 (20.5\%) underwent a sec-

Table 3. Laboratory parameters

\begin{tabular}{lc}
\hline Laboratory parameter & Mean \pm SD (range) \\
\hline WBC $(109 / \mathrm{L})$ & $12.40 \pm 6.35(3.70-36.00)$ \\
$\mathrm{Hb}(\mathrm{g} / \mathrm{dL})$ & $13.30 \pm 1.56(8.90-18.00)$ \\
Platelets $(\mathrm{cmm})$ & $478.20 \pm 84.40(108.00-489.00)$ \\
Albumin $(\mathrm{g} / \mathrm{dL})$ & $3.87 \pm 0.72(1.90-5.20)$ \\
$\mathrm{pH}$ & $7.35 \pm 0.11(6.90-7.54)$ \\
DHL $(\mathrm{U} / \mathrm{L})$ & $343.20 \pm 102.70(167.00-540.00)$ \\
\hline
\end{tabular}

SD, standard deviation; WBC, white blood cells; $\mathrm{Hb}$, hemoglobin; $\mathrm{DHL}$, lactate dehydrogenase. ondary elective procedure in the same hospitalization, a Hartman procedure in 4 patients $(11.7 \%)$, with a colorectal hand-sewn anastomosis 6 months after the procedure in 3 patients $(8.8 \%)$. Fatality occurred in 1 diabetic patient $(2.9 \%)$ on postoperative day (POD) 3 after the initial Hartman procedure due to complications of chronic renal failure, including acute hepatic and respiratory failure. For the remaining 3 patients $(8.8 \%)$ who underwent a secondary procedure after initial decompression, sigmoidectomy with a colorectal hand-sewn anastomosis was achieved. Of the 5 patients who did not undergo a secondary procedure, $3(8.8 \%)$ died (one diabetic and one hypertensive) within 24-48 hours after the initial decompression due to metabolic acidosis and electrolyte disequilibrium secondary to intestinal obstruction. The other 2 patients (5.8\%) were discharged for elective sigmoidectomy with primary anastomosis, but they were lost to follow-up.

The 22 patients $(64.7 \%)$ who did not receive derotation or decompression underwent immediate surgery, with 16 patients (47\%) undergoing a Hartman procedure. Fatality occurred in 3 patients with no comorbidities due to septic shock caused by peritonitis from colonic perforations on POD 3, 5, and 8, respectively. Nine patients $(26.4 \%)$ underwent a colorectal hand-sewn anastomosis 4 months after the Hartman procedure. The remaining 4 patients (11.7\%) were lost to follow-up. A sigmoidectomy with colorectal hand-sewn anastomosis was conducted in 3 patients $(8.8 \%)$ on the initial procedure.

Of the remaining 3 patients $(8.8 \%)$ who underwent emergent surgery $(\mathrm{n}=22)$, cecal volvulus was encountered, for which 2 patients $(5.8 \%)$ underwent a right hemicolectomy with ileo-trans-

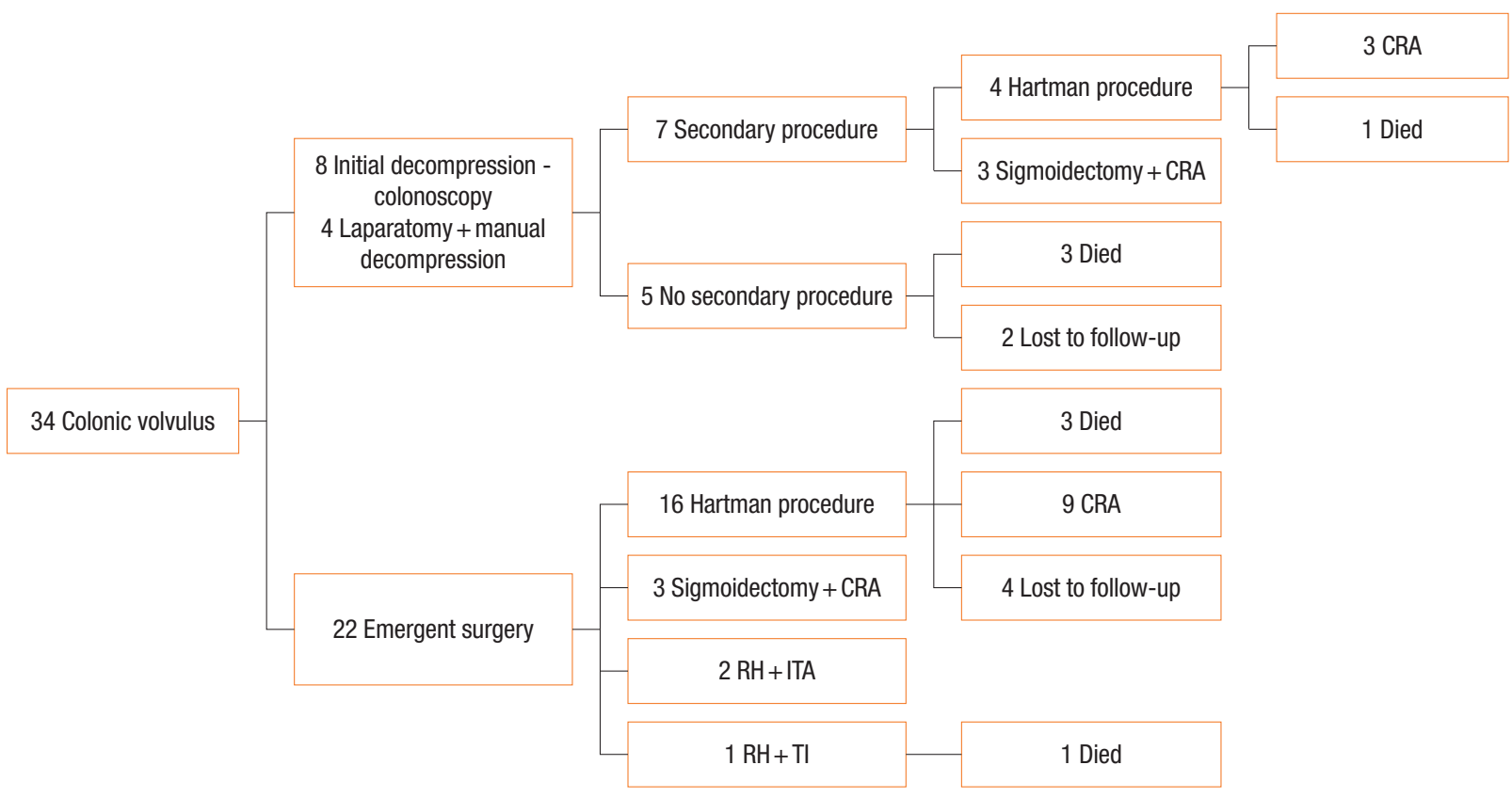

Fig. 1. Patient treatment with outcome. CRA, colorectal anastomosis; IT, ileo-transverse anastomosis; TI, terminal ileostomy; RH, right hemicolectomy. 
Table 4. Patient outcomes

\begin{tabular}{lc}
\hline Outcome & No. (\%) \\
\hline Currently on follow-up & $19(55.8)$ \\
Died from colonic volvulus & $8(23.5)$ \\
Lost to follow-up & $6(17.6)$ \\
Died of breast cancer & $1(2.9)$ \\
\hline
\end{tabular}

verse hand-sewn anastomosis. In the remaining patient $(2.9 \%)$ with known cardiac failure, a right hemicolectomy with a terminal ileostomy and a mucous fistula was achieved. He died on POD 1 due to myocardial infarction. Overall mean blood loss was $98.4 \pm 73.5 \mathrm{~mL}$, and mean operative time was $99.4 \pm 30$ minutes. Patient treatments and outcomes are listed in Fig. 1.

Six patients were lost to follow up, 2 of them (5.8\%) had undergone only initial decompression, and 4 of them (11.7\%) underwent a Hartman procedure and did not return for follow-up. One patient who underwent a Hartman procedure with later anastomosis died of breast cancer 2 years after the reconnection. Nineteen patients (55.8\%) had a 14.3 month mean follow-up, all of them evacuating via the anorectum without complications (Table 4).

\section{DISCUSSION}

In our study, we had only one fatality in our 7 electives operations and 4 fatalities with our 22 emergent procedures, equivalent to the series of Anderson and Lee [11], with a 15\% mortality rate, for elective surgery and a lower mortality rate for emergent surgery compared to that previous study (44\%). Our other 3 fatalities occurred after initial decompression due to metabolic acidosis and electrolyte disequilibrium. We saw an average of 8.5 patients per year with this condition at our hospital. The male: female ratio was $1: 1$, supporting some series in which colonic volvulus affects males and females in nearly equal proportion [11, 13]. Some other series including only sigmoid volvulus showed a predominance in men $[2,3,6,7]$. On the other hand, female sex is more predominant in cecal volvulus in some series [5, 14-17]. In the present study, 2 of 3 patients with cecal volvulus were men.

The average age in our series was 55 years (range, 19-87 years), comparable to some series in which the mean age was 55-65 years [10]. In contrast, some African series had a mean age of 3545 years. This can be explained by the remarkably long and thin mesentery typical of Africans [18]. This expedites a more complex rotation and tighter strangulation at the basis [1, 18-21]. Other factors that may predispose to a sigmoid volvulus include diabetes, neurological and psychiatric diseases, high fiber diet, postoperative adhesions, internal herniations, intussusceptions, omphalomesenteric abnormalities, and intestinal malrotations [2, $3,7,12,17,22]$. In our series, $17.6 \%$ of patients had a psychiatric illness, and $8.8 \%$ had diabetes mellitus.

Furthermore, several factors may also cause a mobile cecum, predisposing to a colonic volvulus; for instance, after a laparoscopic procedure with the use of pneumoperitoneum, when mobilizing the right colon, or after colonoscopy since air insufflation dilates all parts of the colon $[1,23,24]$. Other conditions that may cause a mobile cecum include previous pelvic surgery and pregnancy because the gravid uterus protrudes from the uterine cavity, displacing the cecum [25]. We could not find any predisposing factors that could have explained this emergent condition in our 3 patients.

Despite progress in the treatment of this disease, the optimal surgical technique has not been determined [3, 10, 12, 26, 27]. Emergency surgery is the appropriate treatment for those with acute presentation (diffuse peritonitis, intestinal perforation, or ischemic necrosis), and resective surgery with or without stoma is the most commonly performed surgical procedure in this setting [1]. In their prospective randomized trial in emergent surgery for colonic volvulus, Bagarani et al. [18] concluded that the therapeutic approach to sigmoid volvulus should be diversified according to the absence or presence of gangrenous colon, with primary anastomosis the treatment of choice in patients with viable colon and Hartmann's procedure for patients with peritoneal inflammation suggestive of a gangrenous colon. Hartmann's procedure may be advisable also when compromised mucosa is seen on endoscopy or if the fluid recovered from the obstructed segment is bloody [12]. Because endoscopy equipment was not always available in our hospital, we performed 22 emergent surgeries, 16 of which involved Hartmann's procedure. Ironically, almost all of these patients had a gangrenous colon, and 3 had perforation with fecal peritonitis. In patients with viable colon, we performed 3 sigmoid resections with primary anastomosis with satisfactory results.

Sigmoid volvulus is easily recognized on plain abdominal radiograph, with marked distention of an ahaustral sigmoid colon and an omega loop or coffee bean sign, pathognomonic for this entity $[2,8,12,27]$. Such signs were noted in $76.4 \%$ of our patients. A study of Burrel et al. [28] analyzing radiographic signs for sigmoid volvulus established that an apex of the colonic loop located under the left hemidiaphragm, inferior convergence on the left, and the left flank overlap sign were $100 \%$ specific and highly sensitive for diagnosis of sigmoid volvulus. This can be compared with some series in which the signs were compatible in $80 \%-82 \%$ of radiographs $[12,27]$.

In cases in which the radiographic findings on abdominal Xrays are unclear, a contrast enema may be used because of its high sensitivity for detecting this condition. We did not use contrast enema because almost all our patients were diagnosed with plain films or CT scan [8]. In the latter circumstance, the whirl sign located to the right of midline or in the midline/left is well recognized as a sign of cecal or sigmoid volvulus, respectively, indicating twisting of the mesenteric vessels, fat, and bowel. Nevertheless, its sensitivity and specificity have not been assessed [2, 8, 29]. In our series, we observed this sign in $52.9 \%$ of patients, but only 
$58.8 \%$ underwent CT scan, an accuracy of $90 \%$. In a series of Levsky et al. [8], the whirl sign was present in only 12 of 21 cases (57\%), and in the series of Lou et al. [2], this sign was positive in $100 \%$ of their patients, comparable to our series.

The classic clinical presentation is a triad of abdominal pain, distention, and constipation $[2,8]$, with the first 2 symptoms present in almost all of our patients and the latter present in only $7 \mathrm{pa}-$ tients. In our series, we also found nausea and vomiting to be a common symptom, present in almost half of the patients, whereas diarrhea and melena were only present in $23.5 \%$ of patients.

Late admission is generally a problem in colonic volvulus, and the mean symptom duration is reported to vary between one and 7 days and is more frequently delayed in geriatric patients $[2,6,7$, $11,30]$. This compares to our series, where the interval between development of symptoms and hospitalization ranged from 1 to 14 days, with an average of 5.67 days. Our high mortality rate may be explained by delay in seeking medical attention. Most fatalities were due to acute renal failure due to intestinal obstruction and dehydration, with subsequent perforations concluding with severe peritonitis and abdominal sepsis.

In conclusion, this series contributes to the existing literature on colonic volvulus. Distinctive features of each patient determine the appropriate treatment for this condition, with the main objective of preventing strangulation, perforation, and subsequent abdominal sepsis. This disorder must be suspected when a patient presents with abdominal pain, abdominal distension, and bean sign on plain X-rays and/or a whirl sign on CT scan.

\section{CONFLICT OF INTEREST}

No potential conflict of interest relevant to this article was reported.

\section{REFERENCES}

1. Halabi WJ, Jafari MD, Kang CY, Nguyen VQ, Carmichael JC, Mills S, et al. Colonic volvulus in the United States: trends, outcomes, and predictors of mortality. Ann Surg 2014;259:293-301.

2. Lou Z, Yu ED, Zhang W, Meng RG, Hao LQ, Fu CG. Appropriate treatment of acute sigmoid volvulus in the emergency setting. World J Gastroenterol 2013;19:4979-83.

3. Safioleas M, Chatziconstantinou C, Felekouras E, Stamatakos M, Papaconstantinou I, Smirnis A, et al. Clinical considerations and therapeutic strategy for sigmoid volvulus in the elderly: a study of 33 cases. World J Gastroenterol 2007;13:921-4.

4. Grossmann EM, Longo WE, Stratton MD, Virgo KS, Johnson FE. Sigmoid volvulus in Department of Veterans Affairs Medical Centers. Dis Colon Rectum 2000;43:414-8.

5. Ballantyne GH, Brandner MD, Beart RW Jr, Ilstrup DM. Volvulus of the colon. Incidence and mortality. Ann Surg 1985;202:83-92.

6. Turan M, Sen M, Karadayi K, Koyuncu A, Topcu O, Yildirir C, et al. Our sigmoid colon volvulus experience and benefits of colo- noscope in detortion process. Rev Esp Enferm Dig 2004;96:32-5.

7. Oren D, Atamanalp SS, Aydinli B, Yildirgan MI, Başoğlu M, Polat $\mathrm{KY}$, et al. An algorithm for the management of sigmoid colon volvulus and the safety of primary resection: experience with 827 cases. Dis Colon Rectum 2007;50:489-97.

8. Levsky JM, Den EI, DuBrow RA, Wolf EL, Rozenblit AM. CT findings of sigmoid volvulus. AJR Am J Roentgenol 2010;194: 136-43.

9. Nay HR, West JP. Treatment of volvulus of the sigmoid colon and cecum. Arch Surg 1967;94:11-3.

10. Kuzu MA, Aslar AK, Soran A, Polat A, Topcu O, Hengirmen S. Emergent resection for acute sigmoid volvulus: results of 106 consecutive cases. Dis Colon Rectum 2002;45:1085-90.

11. Anderson JR, Lee D. The management of acute sigmoid volvulus. Br J Surg 1981;68:117-20.

12. Welch GH, Anderson JR. Acute volvulus of the sigmoid colon. World J Surg 1987;11:258-62.

13. Khoury GA, Pickard R, Knight M. Volvulus of the sigmoid colon. Br J Surg 1977;64:587-9.

14. Pahlman L, Enblad P, Rudberg C, Krog M. Volvulus of the colon. A review of 93 cases and current aspects of treatment. Acta Chir Scand 1989;155:53-6.

15. Ruiz-Tovar J, Calero Garcia P, Morales Castineiras V, Martinez Molina E. Caecal volvulus: presentation of 18 cases and review of literature. Cir Esp 2009;85:110-3.

16. Perrot L, Fohlen A, Alves A, Lubrano J. Management of the colonic volvulus in 2016. J Visc Surg 2016;153:183-92.

17. Rodriguez-Wong U, Badillo Bravo A, Cruz Reyes JM, Rovelo Lima E. Volvulo de sigmoides en el Hospital Juarez de Mexico: 20 anos de experiencia. Rev Mex coloproctologia. Rev Mex Coloproctol 2006;12: 112-6.

18. Bagarani M, Conde AS, Longo R, Italiano A, Terenzi A, Venuto G. Sigmoid volvulus in west Africa: a prospective study on surgical treatments. Dis Colon Rectum 1993;36:186-90.

19. Nuhu A, Jah A. Acute sigmoid volvulus in a West African population. Ann Afr Med 2010;9:86-90.

20. Madiba TE, Haffajee MR. Sigmoid colon morphology in the population groups of Durban, South Africa, with special reference to sigmoid volvulus. Clin Anat 2011;24:441-53.

21. Samuel JC, Msiska N, Muyco AP, Cairns BA, Charles AG. An observational study addressing the anatomic basis of mesosigmoidopexy as a rational treatment of non-gangrenous sigmoid volvulus. Trop Doct 2012;42:44-5.

22. Mellor SG, Phillips RK. The aetiology and management of sigmoid volvulus in the UK: how much colon need be excised? Ann R Coll Surg Engl 1990;72:193-5.

23. Agko M, Gociman B, Keilani ZM, Mukherjee A. Cecal volvulus: a rare complication of colonoscopy. Int J Colorectal Dis 2012;27: 265-6.

24. Anderson JR, Spence RA, Wilson BG, Hanna WA. Gangrenous caecal volvulus after colonoscopy. Br Med J (Clin Res Ed) 1983; 286:439-40. 
25. Pal A, Corbett E, Mahadevan N. Caecal volvulus secondary to malrotation presenting after caesarean section. J Obstet Gynaecol 2005;25:805-6.

26. Sule AZ, Misauno M, Opaluwa AS, Ojo E, Obekpa PO. One stage procedure in the management of acute sigmoid volvulus without colonic lavage. Surgeon 2007;5:268-70.

27. Akcan A, Akyildiz H, Artis T, Yilmaz N, Sozuer E. Feasibility of single-stage resection and primary anastomosis in patients with acute noncomplicated sigmoid volvulus. Am J Surg 2007;193:421-6.

28. Burrell HC, Baker DM, Wardrop P, Evans AJ. Significant plain film findings in sigmoid volvulus. Clin Radiol 1994;49:317-9.

29. Macari M, Spieler B, Babb J, Pachter HL. Can the location of the CT whirl sign assist in differentiating sigmoid from caecal volvulus? Clin Radiol 2011;66:112-7.

30. Oncü M, Piskin B, Calik A, Yandi M, Alhan E. Volvulus of the sigmoid colon. S Afr J Surg 1991;29:48-9. 